patients is restricted. This study was designed to determine the in vitro sensitivity to $\mathrm{LMWH}$ of different reagents by sonoclot analyser, and to determine whether the ACT can be used to monitor LMWH.

Methods This study was performed in vitro. ACT was measured with different reagents (glass beads, celite, and kaolin) on volunteer $(n=30)$ blood samples spiked with increasing concentrations of LMWH (dalteparin, 0.2-1.8 IU/ml). Linear regression analysis was performed to establish a regression equation from different concentration of dalteparin and corresponding ACT values.

Results Analysis of dose-response curves obtained in vitro, an excellent linear relationship was observed between the ACT and dalteparin concentrations for all three reagents $(p<0.01)$. Differences in slope of the regression curves of ACT were observed with all the reagents tested (glass beads $249.7 \mathrm{~s} / \mathrm{IU}$, celite $77.7 \mathrm{~s} / \mathrm{IU}$, and kaolin $59.3 \mathrm{~s} / \mathrm{IU}, \mathrm{p}<0.01)$. Reagents vary widely in their in-vitro sensitivity to dalteparin. In the concentration range of $0.2-1.8 \mathrm{IU} / \mathrm{ml}$, the gaolin reagent was too insensitive to dalteparin, and glass beads was the most suitable reagent for monitoring the anticoagulant effect of dalteparin.

Conclusions Using sonoclot analyser, there was an excellent linear relationship between the ACT and dalteparin concentrations for all the three reagents (glass beads, celite, and kaolin) in vitro. Glass beads may be a suitable reagent of ACT test for monitoring the anticoagulant effect of LMWH.

\section{e0171 THE SENSITIVITY OF NEW REAGENTS FOR LABORATORY MONITORING OF LOW MOLECULAR WEIGHT HEPARIN: AN IN VITRO STUDY}

doi:10.1136/hrt.2010.208967.171

Shi Xu-Bo, Hu Da-Yi, Wang Ji-Yun, Hou Xiao-Xia, Ma Zhi-Min. Department of Cardiology, Beijing Tong Ren Hospital, Beijing, China

Objective Low molecular weight heparin (LMWH) is currently the most commonly used intravenous anticoagulant drugs, but the lack of point of care testing (POCT) limit its applications in patients with severe renal dysfunction and others. The purpose of this study was to explore the sensitivity of new activated clotting time (ACT) reagents for laboratory monitoring of LMWH.

Methods Blood samples collected from 30 healthy volunteers. After taking blood samples, different doses of low molecular weight heparin (dalteparin) were added and the anti-Xa level of final blood samples was $0.1-1.8 \mathrm{IU} / \mathrm{ml}$. ACT and clot rate (CR) were measured with traditional reagent kaolin and new reagent magbar, Linear regression analysis was performed and a regression equation was established between different anti-factor Xa levels and the corresponding ACT, CR values.

Results With dalteparin concentration increased, the ACT values were gradually extended and the CR values were gradually reduced with both two reagents (kaolin and magbar). Analysis of doseresponse curves obtained in vitro, an excellent linear relationship was observed between the ACT and dalteparin concentrations for all two reagents $(p<0.01)$, and an exponential relationship was observed between the CR and dalteparin concentrations $(p<0.01)$. Differences in slope of the regression curves of ACT were observed with the reagents tested (magbar $1097.6 \mathrm{~s} / \mathrm{IU}$ vs kaolin $59.3 \mathrm{~s} / \mathrm{IU}$, $\mathrm{p}<0.01$ ).

Conclusions This in vitro study has shown that the sensitivity of traditional ACT test reagent (kaolin) for laboratory monitoring of dalteparin was poor, and the sensitivity of new ACT test reagents (magbar) for laboratory monitoring of dalteparin increased significantly. The new reagents magbar may be used for bedside monitoring of anticoagulant activity of LMWH.

\section{Basic Science: Experiment Research e0172 EFFECTS OF EPO ON PROLIFERATION OF RABBIT BONE MARROW MESENCHYMAL STEM CELLS}

doi:10.1136/hrt.2010.208967.172

${ }^{1}$ Li Zhengzhang, ${ }^{2}$ Cheng Yingzhang, ${ }^{1}$ Sehn Zhe, ${ }^{1}$ Xue Haoping, ${ }^{1}$ Chen Shi. ${ }^{1}$ Department of Cardiology, GaoYou people's Hospital, Jiangsu, China; ${ }^{2}$ Second Affiliated Hospital of Nanchang University School of Medicine, Nanchang, China

Objective To explore effects of erythropoietin (EPO) on proliferation, activity and cell cycle of rabbit bone marrow mesenchymal stem cells (BMSCs).

Methods A total of four healthy male New Zealand white rabbits aged 6 weeks were supplied by the Experimental Animal Center of Nachang University. Rabbit BMSCs were in vitro isolated by the density gradient centrifugation. Cells were assigned into normal, $2 \mathrm{u} /$ $\mathrm{ml}, 4 \mathrm{u} / \mathrm{ml}, 8 \mathrm{u} / \mathrm{ml}, 16 \mathrm{u} / \mathrm{ml}$ groups. In the normal group, cells received normal culture, without additional EPO stimulation. Following EPO intervention, cell morphology and growth were observed. Cell proliferation was detected by MTT assay. Cell cycle changes were measured by flow cytometry. Cell supernatant was collected to detect mass concentration of matrix metalloproteinase-2 (MMP-2).

Results EPO to intervene BMSCs 3d, cells had no abnormal changes, showing colony growth. With increase EPO concentration, cell absorbance gradually increased, especially in the $16 \mathrm{u} / \mathrm{ml}$ group. $(\mathrm{F}=28.029, \mathrm{p}=0.008)$. Compared with the normal group, cell cycle changed in the $2 \mathrm{u} / \mathrm{ml}, 4 \mathrm{u} / \mathrm{ml}, 8 \mathrm{u} / \mathrm{ml}, 16 \mathrm{u} / \mathrm{ml}$ groups, and proliferation index significantly increased $(p<0.05$ or 0.01$)$. Mass concentration of MMP-2 in supernatant was the lowest in the $16 \mathrm{u} /$ $\mathrm{ml}$ group, and the highest in the normal group. Significant differences were detected among groups $(t=213.21, p<0.001)$.

Conclusion EPO can increase the proliferative ability of BMSCs.

\section{e0173 ISOLATION CULTURE AND IDENTIFICATION OF RAT MSC}

doi:10.1136/hrt.2010.208967.173

${ }^{1}$ Li Xueyuan, ${ }^{2}$ Zhang Yang, ${ }^{1}$ Qi Guoxian. ${ }^{1}$ The First Hospital of China Medical University, Shenyang, Liaoning, China; ${ }^{2}$ The Centrol Affiliated Hospital of Shenyang Medical College, Shenyang, Liaoning, China

Aims To investigate different ways of isolating and culturing rat MSC and different serum concentrations of medium for the best selection. Materials and methods Direct adherence and density gradient centrifugation methods are used in MSC isolation, routine and modicum medium change methods are used in MSC culture. We compared the growth state, cell quantity and population doubling time of MSC under different culturing ways and different serum concentration medium such as $10 \%, 11 \%$ and $15 \%$. We identified cultured MSC in logarithmic growth phase (P3 generation) by cell surface antigen and its inducing differentiation function.

Results 4 methods, which are direct adherent and routinely changing of medium method, direct adherent and modicum medium changing method, density gradient centrifugation and routinely changing of medium method and density gradient centrifugation and modicum medium changing method, are used during MSC isolating and culturing respectively. The cellular average doubling time is $36.0 \pm 0.9 \mathrm{~h}, 23.5 \pm 1.1 \mathrm{~h}, 49.8 \pm 12 \mathrm{~h}$ and $48.0 \pm 0.8 \mathrm{~h}$ respectively There are cellular colonies forming 3 to 10 days after isolation, shaping like whirlpool. From the serum concentration screening experiment, we find that $11 \%$ is the most suitable one for MSC growth. The result of cell surface antigen identification of MSC through immunol histochemistry is CD45 (-), CD90 (+), and CD45 $0.38 \%$, CD90 $98.4 \%$ for positive expression of MSC through flow cytometry. MSC can be successfully induced to differentiate to chondrocyte and lipocyte. 
Conclusions Direct adherent and modicum medium changing method is the best one for MSC isolation and culture. $11 \%$ is the most suitable serum concentration for MSC growth.

\section{e0174 EFFECT OF HIF1A ON PROLIFERATION AND DIFFERENTIATION OF MSC UNDER HYPOXIA CONDITION IN VITRO}

doi:10.1136/hrt.2010.208967.174

${ }^{1}$ Li Xueyuan, ${ }^{2}$ Zhang Yang. ${ }^{1}$ The First Hospital of China Medical University, Shenyang, Liaoning, China; ${ }^{2}$ The Centrol Affiliated Hospital of Shenyang Medical College, Shenyang, Liaoning, China

Aims To investigate the effect of HIF-1a on MSC under hypoxia condition.

Materials and methods We transfected HIF-1a into MSC of P3 generation through liposome 2000, and observed the expression of green fluorescence protein in order to assess transfecting efficiency. G418 was used to screen stable transfected cells, and limited dilution method used for monoclone culture of screened cells. We identified the stable HIF-1a transfected MSC through the cell surface antigen testing. We compared the growth state among stable transfected MSC with HIF-1a, vacant plasmid transfected MSC and untransfected MSC under hypoxia condition, and the expression of HIF-1a mRNA, VEGF mRNA, HIF-1a protein and VEGF protein was tested. Results pcDNA3.0-HIF-1a-eGFP can be successfully transfected into MSC mediated by liposome 2000, with efficiency of $21 \%$. Stable monoclone of transfected MSC can be obtained by G418 screening and limited dilution method. Stable transfected MSCs still reserve the ability of differentiating to chondrocyte and lipocyte. MSCs transfected with pcDNA3.0-HIF-1a-eGFP had lower apoptosis $(p<0.05)$, greater proliferation $(p<0.05)$, and more expression of HIF-1a mRNA, VEGF mRNA, HIF-1a protein, VEGF protein than MSCs transfected with vacant plasmid pcDNA3.0- eGFP and untransfected ones under hypoxia condition.

Conclusions Stable transfected MSC with HIF-1a has a significant high expression of HIF-1a protein, HIF-1a mRNA, VEGF protein and VEGF mRNA under hypoxia condition. HIF-1a could reduce MSC apoptosis and enhance its proliferation under hypoxia condition.

\section{e0175 THE EFFECT OF GHRELIN ON THE REGRESSION OF ATHEROSCLEROSIS PLAQUE IN APOE-/- MICE AORTA}

doi:10.1136/hrt.2010.208967.175

Deng Bin, Xie Xiumei, Fang Li, Chen Xiaobin, Chen Meifang. Department of Cardiology, Xiangya Hospital, Central South University, Changsha, Hunan, China

Objective To observe the effect of ghrelin on reducing the $\mathrm{apoE}^{-1-}$ mice plasma IL-8, MCP-1, TNF $\alpha$ level and the NFKBp65 expression in vascular wall and the regression of atherosclerotic plaque.

Method 8 week $\mathrm{ApoE}^{-/-}$mice were fed with Western style meals, and the same age mice C57BL/6J fed with the same meals as control. In the eighth week, $\mathrm{ApoE}^{-/-}$mice were assigned to ghrelin intraperitoneal injection and saline injection group randomly in the twelfth week.All of the groups had blood drawn from eye sockets, with isolated plasma used to measure IL-8, MCP-1, TNF $\alpha$ by ELISA. Mice were killed for examination with stereomicroscopy and paraffin imbedding for $\mathrm{HE}$ and immunohistochemistry, and frozen section for red oil stain.

Result 1. On stereomicroscopy, HE, oil red stain and image analysis equipment measurement demonstrated no plaque at C57BL/6J mice vessels, and both apoE ${ }^{-/-}$group and $\mathrm{ApoE}^{-/-}+$ghrelin groups had atherosclerosis plaque at vessels $(22.56 \pm 2.2$ vs $32.37 \pm 3.2 \mathrm{p}<0.01)$ 2. Contrast to $\mathrm{C} 57 \mathrm{BL} / 6 \mathrm{~J}$ mice, apoE ${ }^{-1-}$ mice has higher plasma TNF $\boldsymbol{\alpha}$, IL-8, MCP-1level $(28.81 \pm 1.8$ vs $11.5 \pm 0.6, \mathrm{p}<0.05 ; 335 \pm 16.7$ vs
$25.0 \pm 2.0, \mathrm{p}<0.05 ; 78 \pm 5.6$ vs $15.8 \pm 2.0, \mathrm{p}<0.05)$, but $\mathrm{apoE}^{-/}+$ghrelin mice has lower TNF $\alpha$, IL-8, MCP-1 level than $\mathrm{ApoE}^{-1-}$ mice $(15.45 \pm 0.98$ vs $24.5 \pm 1.68, p<0.05 ; 168.32 \pm 8.78$ vs $335 \pm 16.7 p<0.05$; $45.5 \pm 4.5$ vs $78.5 \pm 5.6, \mathrm{p}<0.05)$. 3. Contrast to C57BL/6J mice, apoE ${ }^{-1}$ mice NFKBp65 immunohistochemistry positive cell integral calculus value were increase $(1424.23 \pm 167.80$ vs $6859.68 \pm 675.34, p<0.01)$; ghrelin $+\mathrm{apoE}^{-/-}$mice NFKBp65 immunohistochemistry positive cell integral calculus value was lower than $\mathrm{apoE}^{-/-}$mice (3424.78 \pm 321.6 vs 6859.68 $\pm 675.34, \mathrm{p}<0.01)$, ghrelin can decrease the expression of $\mathrm{NF \kappa Bp} 65$ in apoE $\mathrm{E}^{-1-}$ mice aorta.

Conclusion Ghrelin can inhibit the inflammatory response to decrease $\mathrm{ApoE}^{-1-}$ mice atherosclerosis plaque formation.

\section{e0176 THE EFFECTS OF ROSUVASTATIN ON THE EXPRESSION OF HOMOCYSTEINE-INDUCED EXPRESSION OF MATRIX METALLOPROTEINASE-2 (MMP-2) AND CELL MIGRATION IN RAT VASCULAR SMOOTH MUSCLE CELLS}

doi:10.1136/hrt.2010.208967.176

Yangbo Xing, Hangyuan Guo, Yafei Shi. Department of Cardiology, Shaoxing People Hospital, Shaoxing, China

Objective The aim of this study was to investigate the effects of rosuvastatin on the expression of homocysteine-induced expression of matrix metalloproteinase-2 (MMP-2) and cell migration in rat vascular smooth muscle cells (VSMC).

Methods Cultured rat VSMC were incubated with different concentrations of Hcy and rosuvastatin (Hcy $1000 \mu \mathrm{mol} / \mathrm{l}$ ) in vitro for 24, 48 and $72 \mathrm{~h}$. The expression of MMP-2 was determined by using the methods of gelatin zymography and western blotting. Cultured rat VSMC was incubated with different concentrations of Hcy and rosuvastatin (Hcy $1000 \mu \mathrm{mol} / \mathrm{l}$ ) in transwell for 24, 48 and $72 \mathrm{~h}$. The number of VSMC which transited the membrane represented the aggressivity of VSMC.

Results Hcy $(50 \sim 1000 \mu \mathrm{mol} / \mathrm{l})$ increased the expression and activity of MMP-2 significantly. Incubated with the same concentration of Hcy the expression and activity of MMP- 2 of $72 \mathrm{~h}$ was higher than that of $24 \mathrm{~h}$ and $48 \mathrm{~h}$. Hcy reduced the expression of MMP-2 at the concentration of $5000 \mu \mathrm{mol} / \mathrm{l}$. Rosuvastatin could inhibit the augmentation of homocysteine-induced expression and activity of MMP-2. Hcy $(50 \sim 5000 \mu \mathrm{mol} / \mathrm{l})$ could stimulate the migration of VSMC. Rosuvastatin could decrease the stimulation of homocysteine-induced migration of VSMC.

Conclusions These data suggested that Hcy can increase the MMP-2 expression/activity and the migration of VSMC. It may be one of the roles in the pathogenesis of atherosclerosis induced by Hcy. Rosuvastatin can inhibit the augmentation of homocysteineinduced MMP-2 expression/activity and migration of VSMC. This may be one of the pleiotropic of rosuvastatin besides lipid-lowering and benefit the therapy of CHD.

\section{e0177 EXPLORATION NEW METHODS FOR ESTABLISHMENT OF PORCINE MODEL OF ACUTE MYOCARDIAL INFARCTION}

doi:10.1136/hrt.2010.208967.177

Siming Tao, Tao Guo, Shunhua Pu, Zhuo Yu. The First Affiliated Hospital of Kunming Medical College, Kunming, China

Objective To explore and develop one optimise method that it could establish the porcine model of acute myocardial infraction more safer, quicker, convenient than routine methods.

Methods 30 animals with health condition, mean weight $26.5 \pm 4.8 \mathrm{~kg}$; The pigs were divided into two groups randomly, group A ( $\mathrm{n}=13)$ and group $\mathrm{B}(\mathrm{n}=17)$, according to different method. Angioplasty balloon was positioned in the mid-distal of left anterior 